\title{
THE FIVE DIMENSIONAL POLYHEDRAL SCHOENFLIES THEOREM
}

\author{
BY RONALD H. ROSEN ${ }^{1}$ \\ Communicated by J. Milnor, March 9, 1964
}

Introduction. The central result of this note is a proof that in the 5 -sphere with its usual piecewise linear structure, a subpolyhedron homeomorphic to $S^{4}$ bounds two topological 5-cells. Some related facts about imbeddings of $S^{n-1}$ in $S^{n}$ are also included. Our information about such imbeddings is quite incomplete, so it may be appropriate, after making a few definitions, to summarize current knowledge with regard to this problem.

We shall adhere to the notation of [5] and [14]. An imbedding $h$ of $S^{k}$ in $S^{n}$ will be called flat if $\left(S^{n}, h\left(S^{k}\right)\right) \approx\left(S^{n-k-1} \circ S^{k}, S^{k}\right)$; it will be called weakly flat if $S^{n}-h\left(S^{k}\right) \approx S^{n-k-1} \circ S^{k}-S^{k}$. According to classical results every imbedding of $S^{n-1}$ in $S^{n}$ is flat if $n \leqq 2$. M. Brown's recent characterization [4], [5] is that an $(n-1)$-sphere is flat in $S^{n}$ if and only if it is locally flat.

A polyhedron will be called a piecewise linear n-sphere or $n$-cell if it is piecewise linearly equivalent to $\mathrm{Bd} \sigma^{n+1}$ or $\sigma^{n}$, respectively. A combinatorial n-manifold (with boundary) is an $n$-polyhedron in which the link of each $k$-simplex is a piecewise linear $(n-k-1)$-sphere (or a piecewise linear $(n-k-1)$-cell). A star $n$-manifold (with boundary) is an $n$-polyhedron in which the link of each $k$-simplex is a topological $(n-k-1)$-sphere (or a topological $(n-k-1)$-cell). When $n \leqq 4$, these last two notions are equivalent. Newman [12] showed that if $\Sigma$ is an $(n-1)$-sphere which is a subpolyhedron of a star triangulation of $S^{n}$ and $\Sigma$ is itself a star manifold (under the induced triangulation), then $\Sigma$ is flat. Alexander proved in [2] that if $\Sigma$ is an $(n-1)$-sphere which is a subpolyhedron of a piecewise linear $n$-sphere and the closure of one of $\Sigma$ 's complementary domains is a piecewise linear $n$-cell, then the closure of the other complementary domain is a piecewise linear $n$ cell as well. ${ }^{2}$

This brings us to what may be termed the polyhedral Schoenflies conjecture: Suppose an $(n-1)$-sphere $\Sigma$ is a subpolyhedron of the piecewise linear $n$-sphere; must $\Sigma$ be flat? If $n=3$, from work of

1 The author is a National Science Foundation postdoctoral fellow. The author also acknowledges several helpful conversations with D. R. McMillan.

${ }^{2}$ M. H. A. Newman actually established this theorem by more complicated methods in a somewhat earlier series of papers; see especially, On the foundations of combinatory analysis situs, Proc. Roy. Acad. Amsterdam 29 (1926), 610-641. 
Alexander [1] and Moise [10] we know that $\Sigma$ actually bounds two piecewise linear 3-cells. If $n=4$, an affirmative answer is supplied by Newman's theorem [12], since Moise's Three-dimensional Hauptvermutung Theorem [10] implies that $\Sigma$ is a piecewise linear 3 -sphere. Below we shall settle the question for $n=5$ but nothing is as yet known when $n>5$.

One further result ought to be mentioned. Cantrell has established [6] that if $\Sigma$ is an $(n-1)$-sphere in $S^{n}, p$ is a point of $\Sigma, n>3$ and $\Sigma-p$ is locally flat in $S^{n}-p$, then $\Sigma$ is flat. This is of great interest since the corresponding proposition is false for $n=3$. Some further developments in this direction are discussed in §II.

I. Polyhedral 4-manifolds in combinatorial 5-manifolds. If $S^{k} \circ X \approx S^{n+k+1}$ it follows, for example, from Smith theory that $X$ is a generalized $n$-manifold and a homology $n$-sphere. Accordingly for $n=1$ or 2, reference to Wilder's book [16] allows us to conclude that $X \approx S^{n}$; if $n=0, X$ is obviously a 0 -sphere. If $X$ is a polyhedron we may use more elementary arguments to derive the latter relations by employing the formula $\operatorname{Lk}(K, \sigma \circ \tau)=\mathrm{Lk}(\operatorname{Lk}(K, \sigma), \tau)$, where $\sigma \circ \tau$ is a simplex of the polyhedron $K$; in that case $X=\operatorname{Lk}\left(S^{n+k+1}, \sigma^{k}\right)$ for any $k$-simplex $\sigma^{k}$ of $S^{k}$. Hence suppose $M^{m}$ is a triangulated $m$ manifold and $\sigma^{m-1}$ is one of its simplexes; $\operatorname{Lk}\left(M^{m}, \sigma^{m-1}\right)$ is a pair of points. Therefore for $\sigma^{m-2} \subseteq \sigma^{m-1}, \operatorname{Lk}\left(M^{m}, \sigma^{m-2}\right)$ is a closed 1-manifold and thus, a circle. Similarly for $\sigma^{m-3} \subseteq \sigma^{m-2}, \operatorname{Lk}\left(M^{m}, \sigma^{m-3}\right)$ is a closed 2-manifold; since it is a homology 2-sphere, it is in fact a 2-sphere. On the other hand if $\sigma^{m-4} \subseteq \sigma^{m-3}$, we merely know that $\operatorname{Lk}\left(M^{m}, \sigma^{m-4}\right)$ is a combinatorial 3-manifold. More generally $\operatorname{Lk}\left(M^{m}, \sigma^{k}\right)$ is a closed $(n-k-1)$-pseudomanifold. In [13], however, we showed that $\operatorname{Lk}\left(M^{m}, \sigma^{k}\right) \circ S^{k} \approx S^{m}$.

If $S(X) \approx S^{n+1}$ we shall call $X$ a suspension $n$-sphere. If $n>1$, it is not difficult to see that $X$ is simply connected. An imbedding $h$ of $X$ in $S^{n+1}$ shall be designated flat if $\left(S^{n+1}, h(X)\right) \approx(S(X), X)$ and weakly flat if $S^{n+1}-h(X) \approx S(X)-X$ (which is the union of two disjoint open $n$-cells by Theorem 4 of [13]). The only nontrivial examples of suspension spheres are, so far as known, neither manifolds nor polyhedra; the first such example was constructed by Bing from a decomposition of $S^{3}[3]$.

Lemma 1. Let $X$ be a suspension n-sphere and $h$ a homeomorphism of $X \times I$ into $S^{n+1}$. Then $h(X \times 1 / 2)$ is flat.

This was stated without proof as Lemma 6 of [14]; we have been told by $M$. Brown that it has been proved independently by $\mathrm{E}$. Michael. 
Let $0 \leqq t_{1}<t_{2} \leqq 1$. The quotient space $X \times\left[t_{1}, t_{2}\right]$ with $X \times t_{1}$ and $X \times t_{2}$ identified as distinct points, is equivalent to $S(X) \approx S^{n+1}$. Consequently there is a bicollared $n$-sphere $\Sigma$ in $X \times\left[t_{1}, t_{2}\right]$ separating $X \times t_{1}$ from $X \times t_{2}$. Let $U$ and $V$ be the complementary domains of $h(X \times I)$. It may now be seen that $\bar{U}$ and $\bar{V}$ are cellular. Squeezing them to points we notice that the quotient space is equivalent to $S(X)$. From Theorem 1 of [4] it follows that the quotient space may be realized by a map of $S^{n+1}$ onto itself which leaves $h(X \times 1 / 2)$ fixed.

Lemma 2. Suppose $T$ is a combinatorial triangulation of $S^{4}$ and $X$ is a suspension 3-sphere which is a subpolyhedron with respect to $T$. Then $X$ is flat in $S^{4}$.

We have previously observed that $X$ must be a combinatorial 3manifold. Since $X$ is a homology 3 -sphere it is two-sided in $S^{4}$, and so by Theorem 6 of [5] it is bicollared. Consequently by Lemma 1, $X$ is flat. ${ }^{3}$

THEOREM 1. If $M$ is a star 5-manifold and $N$ is 4-manifold which is a subpolyhedron of $M, N$ is locally flat in $M$.

Let $v$ be a vertex of $N$. Consider the link pair $\Sigma^{4}=\mathrm{Lk}(M, v)$ and $X=\mathrm{Lk}(N, v) . \Sigma^{4}$ is a combinatorial 4 -manifold homeomorphic to $S^{4}$ and $X$ is a suspension 3 -sphere lying in $\Sigma^{4}$. Denote by $U$ and $V$ the complementary domains of $X$ in $\Sigma^{4}$. Clearly $\operatorname{Cl}(M, v)=\bar{U} \circ v \cup \bar{V} \circ v$, while $\bar{U} \circ v \cap \bar{V} \circ v=X \circ v=\mathrm{Cl}(N, v)$. By Lemma $2, \bar{U} \approx C(X)$ $=X \circ p$ and so we have $\bar{U} \circ v \approx X \circ(p \circ v) \approx X \circ I \approx q \circ S(X) \approx C\left(S^{4}\right)$ $\approx I^{5}$. By symmetry $\bar{V} \circ v \approx I^{5}$ and it may be concluded that $N$ is locally flat.

Corollary. If $M \approx S^{5}$ and $N \approx S^{4}, N$ is flat in $M$.

This clearly contains the theorem stated in the introduction.

II. A strengthening of a result of McMillan. Suppose $N$ is a manifold imbedded in another manifold $M$. The set $K$ of points at which $N$ fails to be locally flat in $M$ will be termed its singular set. It should be noticed that $K$ is necessarily closed in $N$.

${ }^{3}$ It should be remarked that if the Poincare conjecture is solved in the affirmative, then $X$ would be a 3 -sphere, similarly it would follow that any triangulation of a 4-manifold would automatically be combinatorial. In this case, then, our Theorems 1 and 3 would be trivially true, but our techniques might possibly be applied to higher dimensional Schoenflies problems. 
Lemma 3. Let $K$ be a compact countable set in $E^{n}$. Then $K$ lies on a tame arc.

For $n=1$ this is obvious. For $n=2$ this is a corollary to a wellknown theorem of plane topology [11, Theorem 123, p. 317]. Hence we assume $n>2$. Let $E^{n-1}$ be a hyperplane in $E^{n}$ and $\Pi$, the orthogonal projection of $E^{n}$ on $E^{n-1} ; K^{\prime}=\Pi(K)$ is compact and countable. By induction there is a homeomorphism $h$ of $E^{n-1}$ on itself carrying $K^{\prime}$ into a straight line $L$. We now extend $h$ in the obvious manner to $E^{n-1} \times E^{1}=E^{n}$. Certainly $h(K)$ lies in the 2-dimensional hyperplane $L \times E^{1}$ and we may go back to the case $n=2$.

A direct elementary proof may be given but it is rather longer.

LEMmA 4. Let $\Sigma$ be an $(n-1)$-sphere in $S^{n}$ and $n>3$. If the singular set of $\Sigma$ lies in a set $K$ which is cellular in both $\Sigma$ and $S^{n}$, then $\Sigma$ is weakly flat.

Identifying $K$ to a point with a quotient map $f$ which is one-to-one on $S^{n}-K$, we have $f\left(S^{n}\right) \approx S^{n}$ and $f(\Sigma) \approx \Sigma$. Since $f(\Sigma)$ is locally flat except possibly at the point $f(K)$, by [6] it is flat. Since $S^{n}-\Sigma \approx f\left(S^{n}\right)$ $-f(\Sigma)$, the lemma is established.

TheOREm 2. Suppose $\Sigma$ is an $(n-1)$-sphere in $S^{n}$ with a singular set $K$ which is at most countable and $n>3$. Then $\Sigma$ is weakly flat. [9].

For $n>4$ this is a corollary to a more general result of McMillan

Applying Lemma 3 we see that $K$ lies in an arc $J$ which is tame in $\Sigma$. Obviously $J-K$ is locally flat in $S^{n}-K$. From a theorem of Homma (see p. 92 of [8] and also Gluck's note Restriction of isotopies in the same journal) ${ }^{4}$ and a paper of Cantrell and Edwards [7] it may now be seen that $J$ is tame in $S^{n}$. The last step in the proof is a simple application of Lemma 4.

CoRollary 1 . Let $\varepsilon$ be a closed subset of $E^{n}$ homeomorphic to $E^{n-1}$ with $n>3$; furthermore suppose that the singular set of $\mathcal{E}$ is at most countable. Then $E^{n}-\varepsilon \approx E^{n} \times S^{0}$.

Taking the one point compactification of the pair $\left(E^{n}, \varepsilon\right)$ one gets a pair satisfying the conditions of the theorem.

Corollary 2. Suppose $X$ is a suspension $(n-1)$-sphere and $\mathrm{S}$ is a locally flat $(n-2)$-sphere in $X$. It follows that $S(s)$ is weakly flat in $S(X)$.

4 This has long since appeared in print: T. Homma, On the imbedding of polyhedra in manifolds, Yokohama Math. J. 10 (1962), 5-10. 
If $n>3$, the singular set of $S(s)$ can only contain the suspension vertices. If $n \leqq 3, X$ is an $(n-1)$-sphere and $\delta$ is flat in $X$.

As Cantrell's arguments in [6] show, if we add to the hypothesis of Theorem 2 the stipulation that $\Sigma$ bounds an $n$-cell on one side, it can be proved that $\Sigma$ is flat. One erases an isolated "singular point" $x$ by utilizing the $n$-cell to place a neighborhood of $x$ in $\Sigma-(K-x)$ onto a flat $(n-1)$-sphere. Ultimately one would be forced to the conclusion that $K=\varnothing$. Analogously it may be seen that if one knew $\Sigma$ were flat whenever $K$ contained no more than two points, then this would imply the more general result that $\Sigma$ is flat when $K$ is at most countable (imbed all points of $K$ save one isolated point in an arc tame in $\Sigma)$. These remarks allow us to improve Corollary 2 in the following way: let $U$ and $V$ be the complementary domains of $\delta$ in $X$; if $S(\bar{U})$ $\approx I^{n}$ then also $S(\bar{V}) \approx I^{n}$.

TheOREM 3. Let $T$ be a triangulation of $S^{4}$ and $\Sigma$ a subpolyhedron with respect to $T$ which is homeomorphic to $S^{3}$. Then $\Sigma$ is weakly flat.

Again we consider the link pair $M^{3}=\mathrm{Lk}(T, v)$ and $X=\mathrm{Lk}(\Sigma, v)$ for a vertex $v$ of $\Sigma$. $X$ is locally flat in $M^{3}$, so $X \circ v-v$ is locally flat in $M^{3} \circ v-v$. Therefore the singular set of $\Sigma$ can only contain vertices and we appeal to Theorem 2.

D. R. McMillan has pointed out to the author that the arguments in this section can be adapted to the quite different situation in which the codimension of $\Sigma$ is at least 3 .

Lemma $4^{\prime}$. Let $\Sigma$ be a $k$-sphere in $S^{n}$ and $n-k \geqq 3$. If the singular set of $\Sigma$ lies in a set $K$ which is cellular in both $S^{n}$ and $\Sigma$, then $\Sigma$ is weakly flat.

The proof revolves around showing that $\Sigma^{\prime}=f(\Sigma)$ is flat in $S^{n}$ $=f\left(S^{n}\right)$. It is clear that $\Sigma^{\prime}$ is locally flat except possibly at the point $f(K)$. If $n \geqq 5$ we may quote Stallings [15] to see that $\Sigma^{\prime}$ is flat. For $n=4$ and $k=1$ Homma's theorem and [7] allow us to assert that $\Sigma^{\prime}$ is locally flat; Gluck [8] has shown that in this case $\Sigma^{\prime}$ is flat.

TheOREM $2^{\prime}$. Suppose $\Sigma$ is a $k$-sphere in $S^{n}$ with a singular set which is at most countable. Then if $n-k \geqq 3, \Sigma$ is weakly flat.

This is proved exactly like Theorem 2 except that in the last step we use Lemma $4^{\prime}$.

\section{REFERENCES}

1. J. W. Alexander, On the subdivision of 3-space by a polyhedron, Proc. Nat. Acad. Sci. U.S.A. 10 (1924), 6-8. 320.

2. - The combinatorial theory of complexes, Ann. of Math. 31 (1930), 292- 
3. R. H. Bing, The cartesian product of a certain nonmanifold and a line is $E^{4}$, Ann. of Math. (2) 65 (1957), 484-500.

4. M. Brown, A proof of the generalized Schoenflies theorem, Bull. Amer. Math. Soc. 66 (1960), 74-76.

5. - Locally fat imbeddings of topological manifolds, Ann. of Math. (2) 75 (1962), 331-341.

6. J. C. Cantrell, Almost locally flat embeddings of $S^{n-1}$ in $S^{n}$, Bull. Amer. Math. Soc. 69 (1963), 716-718.

7. J. C. Cantrell and C. H. Edwards, Almost locally polyhedral curves in Euclidean n-space, Trans. Amer. Math. Soc. 107 (1963), 451-457.

8. H. Gluck, Unknotting $S^{1}$ in $S^{4}$, Bull. Amer. Math. Soc. 69 (1963), 91-94.

9. D. R. McMillan, The singular points of a topological embedding, Abstract 63T373, Notices Amer. Math. Soc. 10 (1963), 588.

10. E. E. Moise, Affine structures in 3-manifolds. V, Ann. of Math. (2) 56 (1952), $96-114$.

11. R. L. Moore, Foundations of point set theory, Amer. Math. Soc. Colloq. Publ. Vol. 13, Amer. Math. Soc., Providence, R. I., 1932.

12. M. Newman, On the division of Euclidean $n$-space by topological $(n-1)$ spheres, Proc. Roy. Soc. London 257 (1960), 1-12.

13. R. H. Rosen, Stellar neighborhoods in polyhedral manifolds, Proc. Amer. Math. Soc. 14 (1963), 401-406.

14. - Polyhedral neighborhoods in triangulated manifolds, Bull. Amer. Math. Soc. 69 (1963), 359-361.

15. J. Stallings, On topologically unknotted spheres, Ann. of Math. (2) 77 (1963), 490-503.

16. R. L. Wilder, Topology of manifolds, Amer. Math. Soc. Colloq. Publ. Vol. 32, Amer. Math. Soc., Providence, R. I., 1949.

INSTITUTE FOR AdVANCEd STUdy 